\title{
Sitagliptin attenuates endothelial dysfunction independent of its blood glucose controlling effect
}

\author{
Xin-Miao Chang ${ }^{1,2}$, Fei Xiao ${ }^{3}$, Qi Pan ${ }^{1}$, Xiao-Xia Wang ${ }^{1}$, and Li-Xin Guo ${ }^{1,2, *}$ \\ 'Department of Endocrinology, Beijing Hospital, National Center of Gerontology, Institute of Geriatric Medicine, Chinese Academy of Medical Sciences, \\ ${ }^{2}$ Graduate School of Peking Union Medical College, Chinese Academy of Medical Sciences, ${ }^{3}$ The Key Laboratory of Geriatrics, Beijing Institute of Geriatrics, \\ Beijing Hospital, National Center of Gerontology, National Health Commission, Institute of Geriatric Medicine, Chinese Academy of Medical Sciences, Beijing \\ 100730, P. R. China
}

\section{ARTICLE INFO}

Received February 16, 2021

Revised May 21, 2021

Accepted May 24, 2021

*Correspondence

Li-Xin Guo

E-mail: glxwork2016@163.com

\section{Key Words}

Apoptosis

Autophagy

Human umbilical vein endothelial cell

Sitagliptin phosphate

Streptozotocin diabetes
ABSTRACT Although the contributions of sitagliptin to endothelial dysfunction in diabetes mellitus were previously reported, the mechanisms still undefined. Autophagy plays an important role in the development of diabetes mellitus, but its role in diabetic macrovascular complications is unclear. This study aims to observe the effect of sitagliptin on macrovascular endothelium in diabetes and explore the role of autophagy in this process. Diabetic rats were induced through administration of high-fat diet and intraperitoneal injection of streptozotocin. Then diabetic rats were treated with or without sitagliptin for 12 weeks. Endothelial damage and autophagy were measured. Human umbilical vein endothelial cells were cultured either in normal glucose or in high glucose medium and intervened with different concentrations of sitagliptin. Rapamycin was used to induce autophagy. Cell viability, apoptosis and autophagy were detected. The expressions of proteins in c-Jun $\mathrm{N}$-terminal kinase (JNK)-Bcl-2-Beclin-1 pathway were measured. Sitagliptin attenuated injuries of endothelium in vivo and in vitro. The expression of microtubuleassociated protein 1 light chain 3 II (LC3II) and beclin-1 were increased in aortas of diabetic rats and cells cultured with high-glucose, while sitagliptin inhibited the over-expression of LC3II and beclin-1. In vitro pre-treatment with sitagliptin decreased rapamycin-induced autophagy. However, after pretreatment with rapamycin, the protective effect of sitagliptin on endothelial cells was abolished. Further studies revealed sitagliptin increased the expression of $\mathrm{BCl}-2$, while inhibited the expression of JNK in vivo. Sitagliptin attenuates injuries of vascular endothelial cells caused by high glucose through inhibiting over-activated autophagy. JNK-Bcl-2-Beclin-1 pathway may be involved in this process.

\section{INTRODUCTION}

Type 2 diabetes is accompanied by hyperglycemia-induced endothelial dysfunction that leads to increased risk of fatal cardiovascular events [1]. Several cardiovascular outcomes trials with glucose-lowering incretin-based therapies have recently reported positive effect on cardiovascular health [2], however the underlying mechanisms of how the beneficial effect is achieved in popu- lations with diabetes remain unclear.

Glucagon-like peptide 1 (GLP-1) is one of the most studied incretin, which lowers blood glucose by promoting insulin secretion, inhibiting glucagon secretion, suppressing appetite, and delaying gastric emptying [3]. Importantly, GLP-1 receptors are not restricted to the pancreas; therefore, GLP-1 can cause additional non-glycemic effects, which are not understood well [4]. GLP-1 is rapidly decomposed by dipeptidyl peptidase 4 (DPP-4) [5]. DPP- (i) \$ This is an Open Access article distributed under the terms of the Creative Commons Attribution Non-Commercial License, which permits unrestricted non-commercial use, distribution, and reproduction in any medium, provided the original work is properly cited. Copyright @ Korean J Physiol Pharmacol, pISSN 1226-4512, elSSN 2093-3827
Author contributions: X.M.C., L.X.G., and F.X. designed the experiment. X.M.C., Q.P., and X.X.W. performed the experiment. X.M.C. analyzed the data and wrote the manuscript. All authors read and approved the manuscript. 
4 inhibitor can inhibit DPP-4 activity and achieve hypoglycemic effect by increasing the active GLP-1 concentration and duration of GLP-1 action. Sitagliptin, a commonly used DPP-4 inhibitor, was previously reported to improve endothelium-dependent relaxation and attenuate the endothelial impairment in diabetic models [6-8]. Recent studies focusing on sitagliptin-induced preservation of endothelial cell functions suggested that improvement of diabetic ischaemia angiogenesis and blood perfusion may be mediated by sitagliptin-induced prevention of endothelial cell apoptosis via augmenting autophagy [9]. The observed protective effects of sitagliptin was linked to increased expression of endothelial nitric oxide synthase and microtubule-associated protein 1 light chain 3 (LC3) and decreased the expression of inducible nitric oxide synthase, 3-nitrotyrosine, and p62 [8]. Unexpectedly, these findings suggest a mechanism that sitagliptin treatment promotes autophagy and this drives an accelerated endothelial regeneration process [8]. While the reported phenomenon is tempting, protective role of autophagy in the maintenance of endothelial tissue integrity needs further confirmation.

Investigations have linked the beneficial effects of DPP-4 inhibitors and Sitagliptin to their impact on glucose homeostasis. However, there are also evidence that DPP4-inhibitors may also possess modulatory function via other signaling pathways and playing a role in the immune system [10]. In fact, a membranebound form of DPP-4 is found on non-pancreatic cell types, including endothelial cells [11], T cells [12] and adipocytes [13]. Considering that DPP-4 acts as a regulatory protease for cytokines, chemokines, and neuropeptides involved in inflammation, immunity, and vascular function [13], it is very likely that Sitagliptin can play a protective role in diabetes associated cardiovascular disease via other mechanisms than lowering blood sugar level.

Various rodent models have been established to study immunologic mechanisms and metabolic function in diabetes. Diabetes induced by streptozotocin (STZ) targets $\beta$ cells, resulting in hypoinsulinemia and hyperglycemia [14]. This in vivo model allows exploring non-glycemic effect of Sitagliptin.

Here we set out to investigate the effect of Sitagliptin on macrovascular endothelium in STZ diabetic rat model and to dissect the role of autophagy in this process. Our findings implicate that Sitagliptin has a significant, previously underappreciated effect that is independent of its well-known glucose lowering effect.

\section{METHODS}

\section{Ethics approval and consent to participate}

The study conformed to the Helsinki Declaration of 1975 (as revised in 2013) concerning Animal Rights. The present study was approved by institutional animal care and use committee of Beijing Vital River Laboratory Animal Technology Co., Ltd. (Bei- jing, China).

\section{Animals and cells}

Twenty-four male Sprague-Dawley rats (weight 150-180 g) purchased from Beijing Vital River Laboratory Animal Technology Co. Ltd (Beijing, China) were randomly divided into three groups: Control $(\mathrm{n}=8)$, diabetes mellitus $(\mathrm{DM})(\mathrm{n}=8), \mathrm{DM}+\mathrm{Si}-$ tagliptin $(n=8)$. The rats in Control group were fed with standard diet, while other rats received high-fat (HF) diet (60\% of calories derived from fat, D12492i; Research Diets, New Brunswick, NJ, USA) for 4 weeks. Then HF rats were intraperitoneal injected once with low dose of streptozotocin $(30 \mathrm{mg} / \mathrm{kg}$; Sigma-Aldrich, St. Louis, MO, USA). Blood glucose was tested 1 week after injection. Rats with glucose levels $\geq 16.7 \mathrm{mmol} / \mathrm{L}$ for twice were regarded as DM rats. Rats in sitagliptin group were given sitagliptin (10 mg/kg/day; Merck, Kenilworth, NJ, USA) by gavage for 12 weeks, while rats in control group and DM group were given an equal volume of saline. Weight, fasting blood glucose (FBG) and random blood glucose were measured every week. All the rats were euthanized at the end of seventeenth week. All experiments were approved by the Ethics Committee of Vital River laboratories.

Human umbilical vein endothelial cells (HUVECs) were isolated from fresh human umbilical cords using $0.1 \%$ collagenase I as previously described [15]. The procedure was approved by the Beijing Hospital Ethics Committee. Cells were cultured in M199 medium (Gibco, Grand Island, NY, USA) supplemented with 20\% fetal bovine serum and $1 \%$ penicillin-streptomycin. HUVECs were incubated at $37^{\circ} \mathrm{C}$ with $5 \% \mathrm{CO}_{2} / 95 \% \mathrm{O}_{2}$ and used at passage number 2-4 for experiments. The cells were treated with normal physiological level $(5.5 \mathrm{mmol} / \mathrm{L})$ or high glucose-containing (33 $\mathrm{mmol} / \mathrm{L}$ ) medium for $72 \mathrm{~h}$. In the dose-dependent experiments, cells were exposed to high glucose and treated with increasing concentrations $(0,0.1,1,10$, and $100 \mu \mathrm{mol} / \mathrm{L})$ of sitagliptin (MedChemExpress, Shanghai, China) for $48 \mathrm{~h}$. To dissect the causal relationship between rapamycin-induced autophagy, and the protective role of sitagliptin, we designed two experiments. In the first, we applied rapamycin $(10 \mu \mathrm{mol} / \mathrm{L}$; MedChemExpress) pretreatment for $30 \mathrm{~min}$ before the administration of highglucose and sitagliptin. In the second, we pretreated the cells with sitagliptin prior to the administration of increasing rapamycin concentration.

\section{Tissue collection}

Rats were fasted overnight and anesthetized by intraperitoneal injection of $1 \%$ pentobarbital sodium (50 mg/kg; Sigma-Aldrich). Blood samples were taken from inferior vena cava. Part of thoracic aortas were isolated and fixed in $4 \%$ paraformaldehyde for pathological and immunohistochemical assays. The other part of aortas were frozen in liquid nitrogen and stored at $-80^{\circ} \mathrm{C}$ for 
Western blot. At the end of the experiments, rats were sacrificed by injection of excessive pentobarbital sodium.

\section{Blood sample measurements}

The level of serum FBG, total cholesterol (TC), triglycerides (TG), and low-density lipoprotein cholesterol (LDL-C) were determined by automatic biochemical analyzer (Hitachi, Tokyo, Japan). Serum vascular adhesion molecule-1 (VCAM-1) was measured with enzyme-linked immunosorbent assay kits (Huamei, Wuhan, China) according to the manufacturer's recommendations. All analyses were performed in duplicates.

\section{Hematoxylin and eosin staining (H\&E)}

Following $4 \%$ paraformaldehyde fixation for $48 \mathrm{~h}$, thoracic aorta tissue samples were dehydrated, embedded in paraffin, sectioned ( $5 \mu \mathrm{m}$ thickness), and stained with H\&E. The specimens were observed under a light microscope and the intima-media thickness of aorta walls were quantitatively assessed with ImageJ software.

\section{Terminal dexynucleotidyl transferase (TdT)-mediated dUTP nick end labeling (TUNEL) assay}

TUNEL assay was used to evaluate apoptosis in tissue samples, using the In Situ Cell Death Detection kit (Roche, Mannheim, Germany) according to manufacturer's protocol. Images were captured with a fluorescence microscope. The number of TUNEL positive cells were counted in random three fields of each specimen $(n=8)$ in every treatment group.

\section{Immunohistochemistry analysis}

Sections of aorta tissue samples were blocked with $5 \%$ bovine serum albumin for $30 \mathrm{~min}$. Slides were then incubated with primary antibody against LC3 (1:100; Proteintech, Chicago, IL, USA) overnight at $4^{\circ} \mathrm{C}$ and with secondary antibody (anti-rabbit $\operatorname{IgG}$ ) for $30 \mathrm{~min}$ at $37^{\circ} \mathrm{C}$. Target protein was stained with diaminobenzidine, while cell nuclei were counterstained with hematoxylin. Positively stained cells were brown in color. Photographs were taken by a light microscope.

\section{Cell viability}

Cell viability was determined using MTT assay. Briefly, HUVECs were seeded in 96-well plates at $1 \times 10^{5} / \mathrm{ml}$ and treated as described above. Added $10 \mu \mathrm{l}$ MTT to each well for $4 \mathrm{~h}$ and replaced with $150 \mu \mathrm{l}$ dimethyl sulfoxide. The optical density was read at $568 \mathrm{~nm}$ using a microplate reader.

\section{Flow cytometry}

Cell apoptosis was assessed by Annexin V-fluorescein isothiocyanate (FITC)/propidium iodide (PI) apoptosis detection kit (KeyGEN BioTECH, Nanjing, China) according to the manufacturer's instruction. Cells were labeled with Annexin V and PI for $10 \mathrm{~min}$, and then detected by flow cytometry.

\section{Western blot analysis}

Western blotting was performed as described elsewhere. Briefly, aorta tissues and cells were lysed in RIPA buffer (Solarbio, Beijing, China). Protein samples were separated by $10 \%-12 \%$ SDS-PAGE and transferred to a PVDF membrane, which was then blocked with $5 \%$ fat-free milk. The blots were incubated with primary antibodies against LC3 (1:1,000; Proteintech), Beclin-1 (1:1,000; Proteintech), JNK (1:1,000; Proteintech), $\beta$-actin (1:1,000; Proteintech), E-selectin (1:1,000; Affinity, Cincinnati, OH, USA), intercellular adhesion molecule 1 (ICAM-1) (1:1,000; Affinity), Von Willebrand factor (VWF) (1:1,000; Affinity), Thrombomodulin (TM) (1:1,000; Affinity), lysosomal-associated membrane protein 1 (LAMP1) (1:1,000; Affinity), lysosomal-associated membrane protein 2 (LAMP2) (1:1,000; Affinity) and Bcl-2 (1:1,000; Affinity) at $4^{\circ} \mathrm{C}$ overnight, then followed by incubation with horseradish peroxidase conjugated goat anti-rabbit IgG or goat anti-mouse IgG (1:5,000; Zhongshan Golden Bridge Bio-technology, Beijing, China) for $1 \mathrm{~h}$ at room temperature. Finally, ECL substrate (Thermo Scientific, Waltham, MA, USA) was applied to the blots, and images were captured in a gel documentation system. Target signals were normalized to $\beta$-actin signal and analyzed semiquantitatively with ImageJ software.

\section{ELISA}

ELISA was carried out as previously described. Briefly, ELISA was carried out in 96-well ELISA microplates (Polystyrene; Microlon 200, U-bottom; Greiner Bio-one, Monroe, NC, USA). The pre-coated wells were rinsed twice with $200 \mu \mathrm{l}$ of $1 \times \mathrm{PBS}$, blocked with $10 \%$ skim milk at room temperature (RT) for $1 \mathrm{~h}$, and again rinsed twice with PBS. Samples, diluted at 1:100 in 5\% skim milk, were added at a volume of $100 \mu \mathrm{l} /$ well in triplicates and were incubated at RT for $1 \mathrm{~h}$. The plates were washed 3 times with $200 \mu \mathrm{l}$ of $0.1 \%$ Tween 20 in $1 \times$ PBS $(1 \times$ PBST) to remove unbound primary antibody. For each wash step, the plates were incubated on a rocker for 5 min before the washing solution was discarded. After the three wash steps, the plates were subjected to a final rinse with PBS.

The OD was measured using an ELISA plate reader using dual wavelengths (405 nm and $650 \mathrm{~nm}$ ). 


\section{Statistical analysis}

Statistical analysis was performed using SPSS 22.0 software (IBM Co., Armonk, NY, USA). Measurement data that conformed to the normal distribution were expressed as mean \pm standard deviation (SD). Independent t-test was used for comparison between two groups, one-way ANOVA test was used for comparison between three groups, and repeated measurement data was analyzed by repeated measurement variance analysis. $p$
$<0.05$ was considered statistically significant.

\section{RESULTS}

\section{Sitagliptin does not reduces blood glucose level in streptozotocin-induced diabetic rats}

To validate our diabetic rat model, we assessed blood glucose
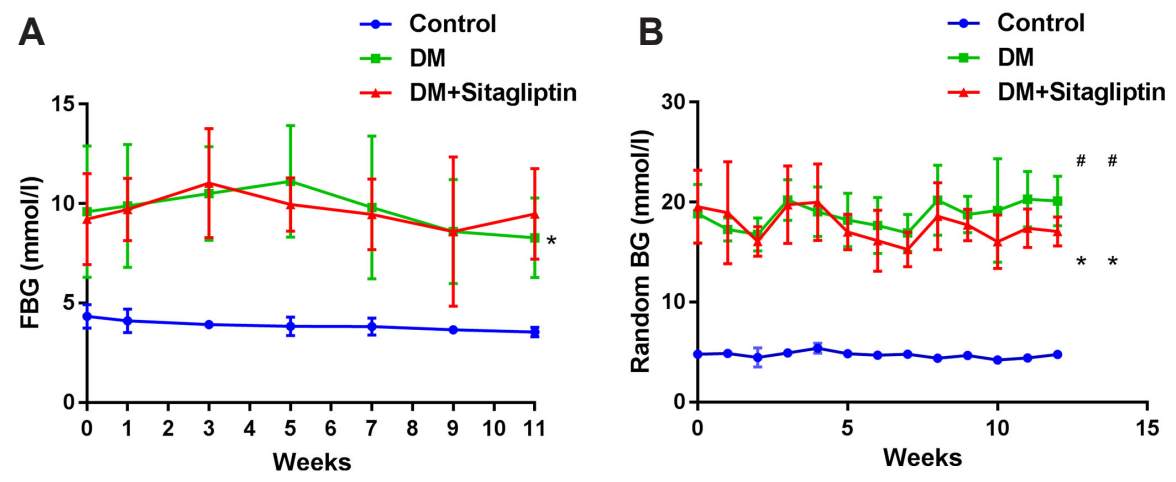

C

Control
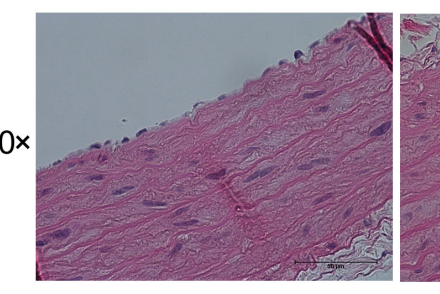

D

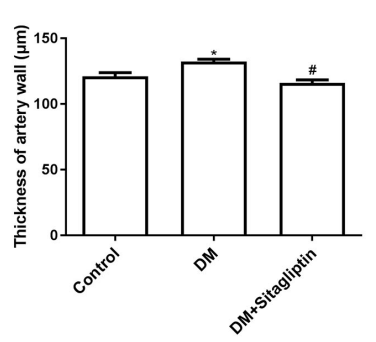

F Control DM DM+Sita

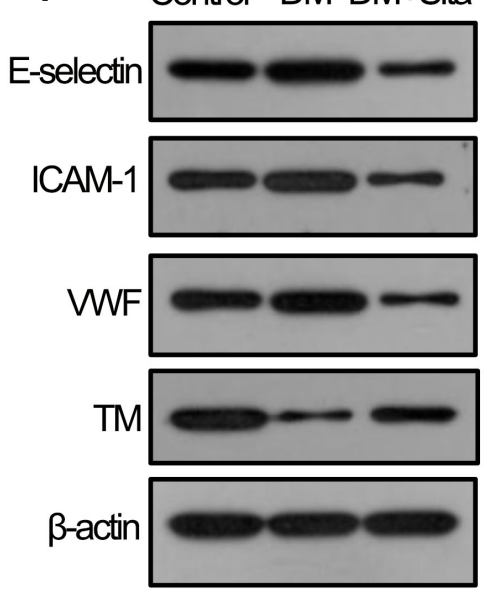

E

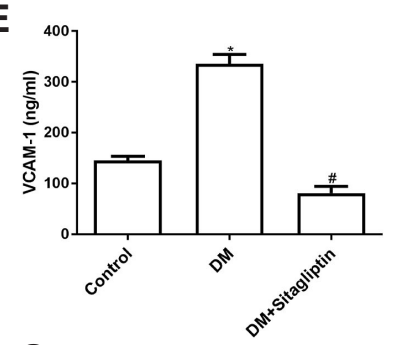

\section{G}

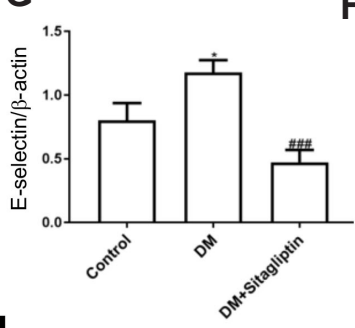

H

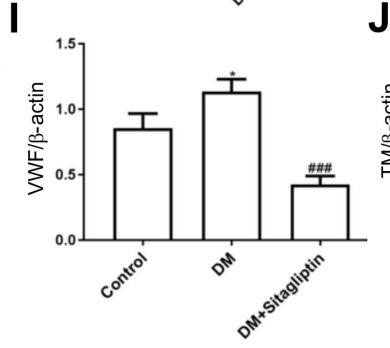

Fig. 1. Sitagliptin improved blood glucose and damage of aortas in diabetic rats. (A) Variation of FBG in the duration of treatments. (B) Variation of random blood glucose in the period of treatments. (C) The arrows point to endothelial cells. H\&E staining of aortas $(\times 200)$. (D) The thickness of artery wall of rats in each group. (E) Serum VCAM-1 level of rats in each group. (F-J) Representative Western blot bands for the protein levels of E-selection, ICAM-1, VWF, and TM in sitagliptin-treated rats. Data are expressed as means $\pm S D, n=5-8$. FBG, fasting blood glucose; $\mathrm{H} \& \mathrm{E}$, hematoxylin and eosin; VCAM-1, vascular adhesion molecule-1; DM, diabetes mellitus; ICAM-1, intercellular adhesion molecule 1; VWF, Von Willebrand factor; TM, thrombomodulin. ${ }^{*} p<0.05$ vs. control group, ${ }^{* * *} p<0.001$ vs. control group, ${ }^{*} p$ $<0.05$ vs. DM group, ${ }^{\# \#} \mathrm{p}<0.001$ vs. DM group. 
and FBG levels, and found that both were higher in diabetic rats compared with the controls. Sitagliptin treatment for 12 weeks did not decrease FBG significantly (Fig. 1A), and random blood glucose level was slightly, but significantly reduced compared to the control diabetic group (Fig. 1B). During the period of treatment, the weight of all the rats was increased, while at the end of treatment, the gain of weight was less in DM + Sitagliptin group than that in DM group, but the difference was not significant (Table 1). Overall, these experiments confirm that Sitagliptin has no blood sugar lowering effect in STZ diabetic models.

\section{Sitagliptin attenuate injuries of aortas in diabetic rats}

Next, we performed H\&E staining to assess the morphological changes in the thoracic aortas. As representative pictures on Fig. 1C shows, in control group the endothelium had smooth and continuous coverage in the elastic membrane. In contrast, the endothelium in diabetic rats was severely damaged, with endothelial cells desquamating from the vascular wall (Fig. 1C). Sitagliptin attenuated the injuries of endothelium in diabetic rats with relatively smooth and intact intima (Fig. 1C). Intima and media thickness of aortas was significantly increased in diabetic rats; however, sitagliptin administration attenuated the impairment of aortic intima structure (Fig. 1D).

In addition to histological evaluation, we also assessed endothelial dysfunction by quantifying molecular markers, such as VCAM-1, ICAM-1, VWF, well-established inflammatory markers mainly secreted when vascular endothelial cells are damaged [16]. The level of serum VCAM-1 in diabetic rats was increased compared to the control group; however in the sitagliptin-treated animals the level of VCAM-1 was comparable to the control group (Fig. 1E). Similarly, ICAM-1 and VWF also showed an increased protein level in the diabetic animals, that was lowered in sitagliptin-treated animals (Fig. 1F-J). In contrast, TM showed lower protein level in diabetic rats (Fig. 1F-J). Overall, the histological and protein-level quantification of pro-inflammatory markers confirmed that sitagliptin can improve endothelium function, even in our STZ diabetic model with hyperglicemia [8].

\section{Sitagliptin inhibited hyperactivation of autophagy in aortas of diabetic rats}

To further explore our finding on the anti-apoptotic property of sitagliptin in the context of previous research documenting increased autophagy of endothelial cells upon sitagliptin-treatment [8], we decided to use similar assays to assess autophagy in our model. First, we measured the expression of LC3 in thoracic aortas. During the formation of autophagosomes, LC3 transform from LC3I to LC3II, and the increased level of LC3II is considered as the most reliable indicator of autophagy [17]. We detected the expression of LC3II by immunohistochemistry (Fig. 2A) and western blot (Fig. 2B, C), both results showed that the amount of LC3II was clearly higher in diabetic rats, while sitagliptin treatment could prevent the elevation in the level of LC3II. To confirm our findings, we also measured the level of beclin-1, an another biomarker of autophagy [18]. We detected increased level of beclin-1 in aortas of diabetic rats, but the level was decreased after sitagliptin intervention, consistent with the results of our LC3 assay (Fig. 2D, E). We further confirmed our conclusions by assessing additional autophagy markers as well, including LAMP1/2 and Cathepsin B/D activity (Fig. 2F).

Thus, our autophagy assays suggest that in vivo sitagliptin inhibit autophagy in aortas of diabetic rats.

\section{Sitagliptin prevent apoptosis of HUVEC cells from high-glucose-induced injuries}

To further evaluate the effect of sitagliptin on cell viability and apoptosis in vitro in a more controlled environment, we set up an in vitro cell culture assay, where we assessed cellular injury caused by high-glucose in HUVECs in the presence or absence of sitagliptin. The viability of HUVECs cultured in high glucose (HG) was decreased compared with that in normal glucose (Fig. 3A; control vs. HG). Supplementation of sitagliptin at a concentration ranging from 0.1 to $100 \mu \mathrm{M}$ increased cell viability in a dosedependent manner. Sitagliptin exerted a most striking protective effect at a concentration of $10 \mu \mathrm{M}$ (Fig. 3A). Number of apoptotic cells was quantitatively assessed by flow cytometry. We found that the total apoptotic rate of HUVECs cultured with high glu-

Table 1. Basic parameters of rats in control, DM, and DM + Sitagliptin groups

\begin{tabular}{lccc}
\hline \multicolumn{1}{c}{ Variable } & Controls $(\mathrm{n}=8)$ & DM $(\mathrm{n}=8)$ & DM + Sitagliptin $(\mathrm{n}=8)$ \\
\hline Body weight $(\mathrm{g})$ & $538.88 \pm 50.16$ & $424.17 \pm 48.02^{*}$ & $380.08 \pm 22.66^{*, *}$ \\
Weight gain $(\mathrm{g})$ & $147.38 \pm 37.53$ & $65.50 \pm 22.04^{*}$ & $50.33 \pm 15.81^{*}$ \\
FBG $(\mathrm{mM})$ & $3.55 \pm 0.24$ & $9.48 \pm 2.28^{*}$ & $8.28 \pm 2.01^{*}$ \\
Random BG $(\mathrm{mM})$ & $4.76 \pm 0.26$ & $20.1 \pm 2.49^{*}$ & $17.07 \pm 1.46^{*, *}$ \\
TC $(\mathrm{mM})$ & $1.00 \pm 0.27$ & $1.44 \pm 0.46^{*}$ & $0.86 \pm 0.28^{*}$ \\
TG $(\mathrm{mM})$ & $0.40 \pm 0.09$ & $0.70 \pm 0.49$ & $0.68 \pm 0.13^{*}$ \\
LDL-C $(\mathrm{mM})$ & $0.17 \pm 0.04$ & $0.18 \pm 0.05$ & $0.11 \pm 0.04^{*, *}$ \\
\hline
\end{tabular}

Values are presented as mean \pm SD. FBG, fasting blood glucose; TC, total cholesterol; TG, triglyceride; LDL-C, low-density lipoprotein cholesterol; DM, diabetes mellitus. ${ }^{*} \mathrm{p}<0.05$ vs. control group; ${ }^{\sharp} \mathrm{p}<0.05$ vs. DM group. 


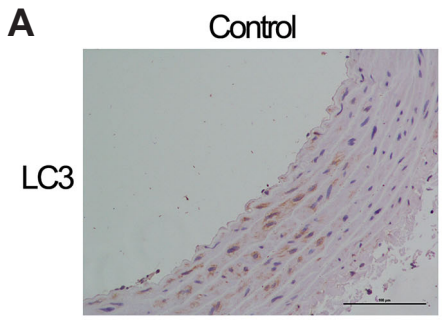

B

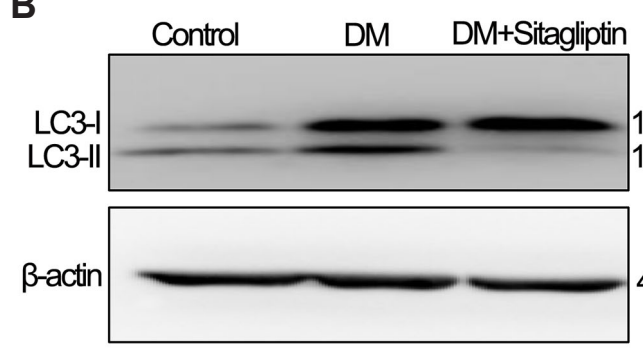

D
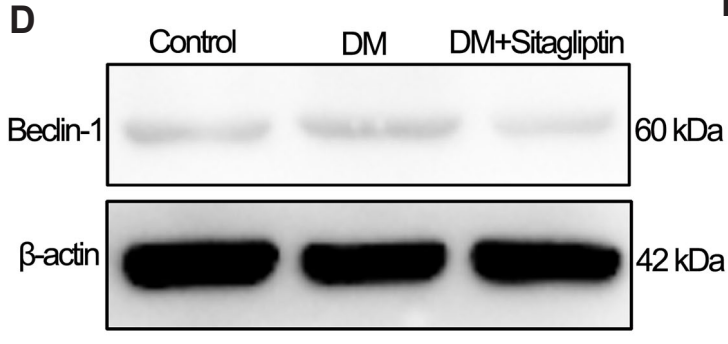

$\mathbf{F}$
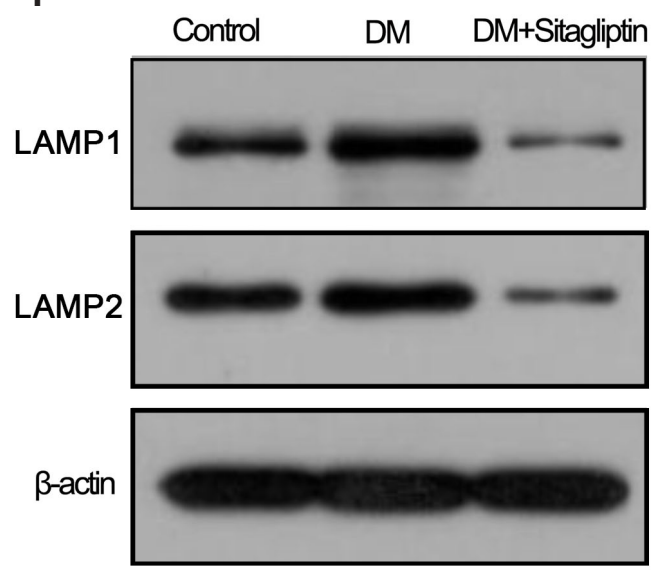

H

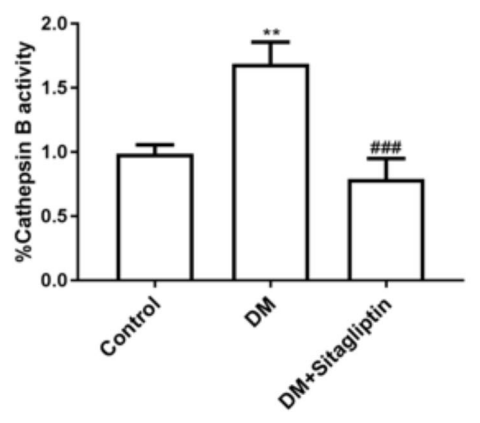

$18 \mathrm{kDa}$ $16 \mathrm{kDa}$

$42 \mathrm{kDa}$

C

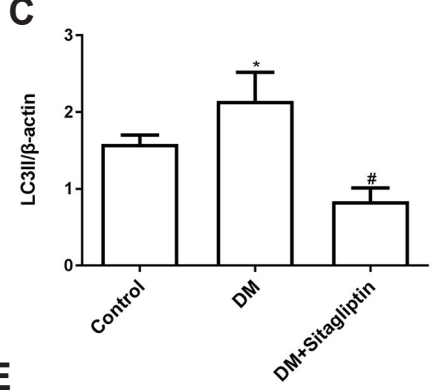

E
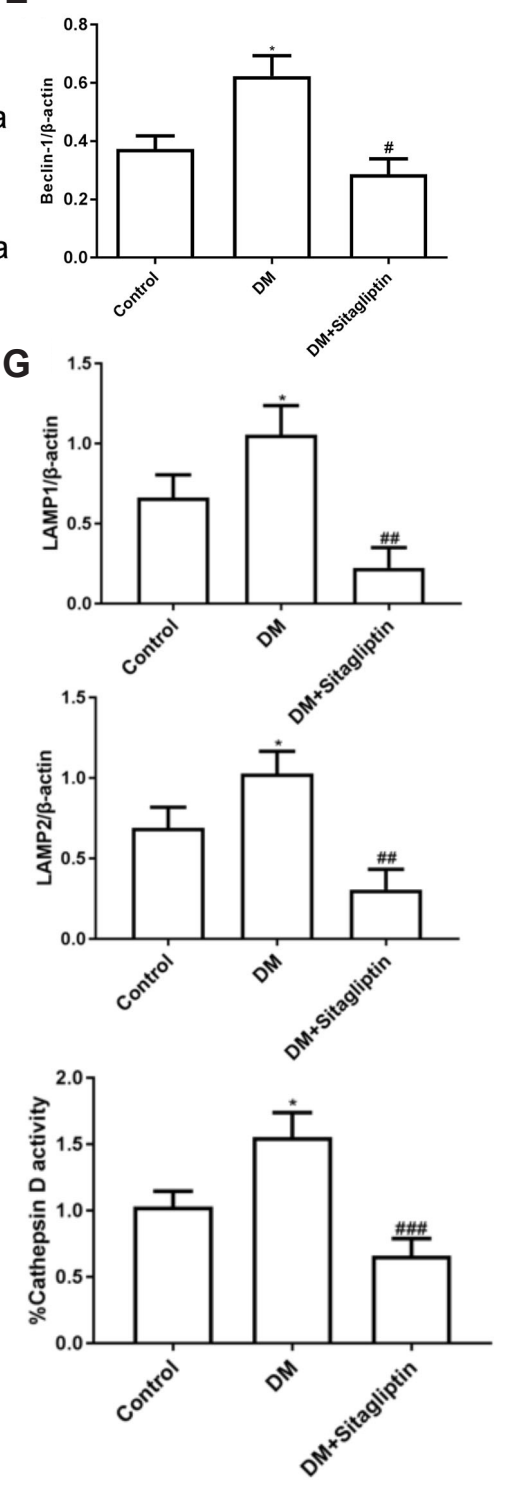

Fig. 2. Sitagliptin inhibited autophagy in aortas in diabetic rats. (A) Representative pictures of immunohistochemistry of LC3 (x200). (B, C) Representative Western blot bands for the protein expression of LC3. (D, E) Representative Western blot bands for the protein expression of beclin-1. $(F, G)$ Representative Western blot bands for the protein expression of LAMP1/2 of aortas in Sitagliptin-treated diabetic rats. $(\mathrm{H})$ The Cathepsin B/D activity results of aortas in Sitagliptintreated diabetic rats were presented. $\mathrm{n}$ = 4-5. LC3, microtubule-associated protein 1 light chain 3; LAMP1/2, lysosomalassociated membrane protein 1/2; DM, diabetes mellitus. ${ }^{*} p<0.05$ vs. control group, ${ }^{* *} p<0.01$ vs. control group, ${ }^{*} p$ $<0.05$ vs. DM group, ${ }^{\# \#} \mathrm{p}<0.01$ vs. DM group, $^{\# \# \#} \mathrm{p}<0.001$ vs. DM group. 
A
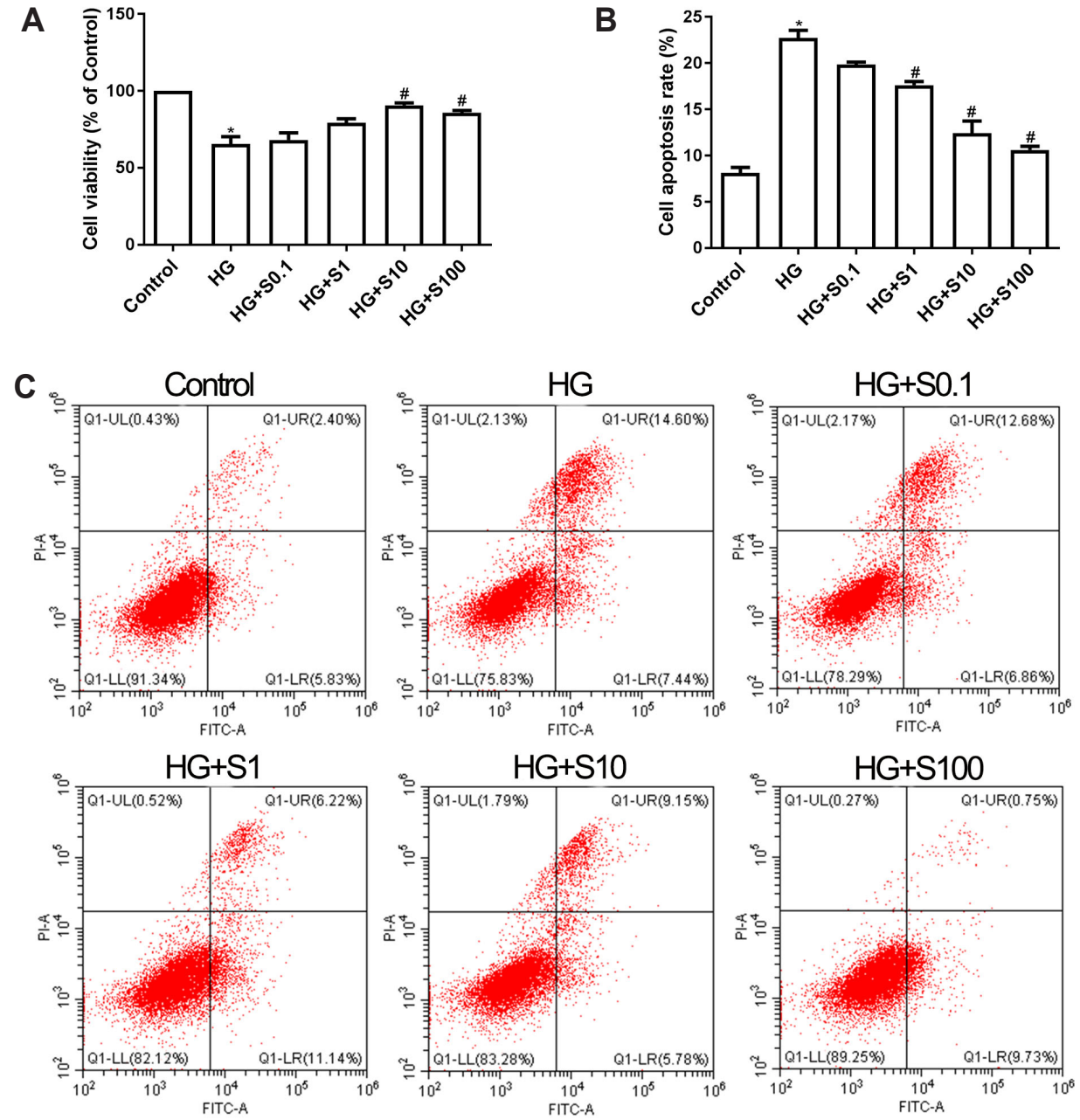

D
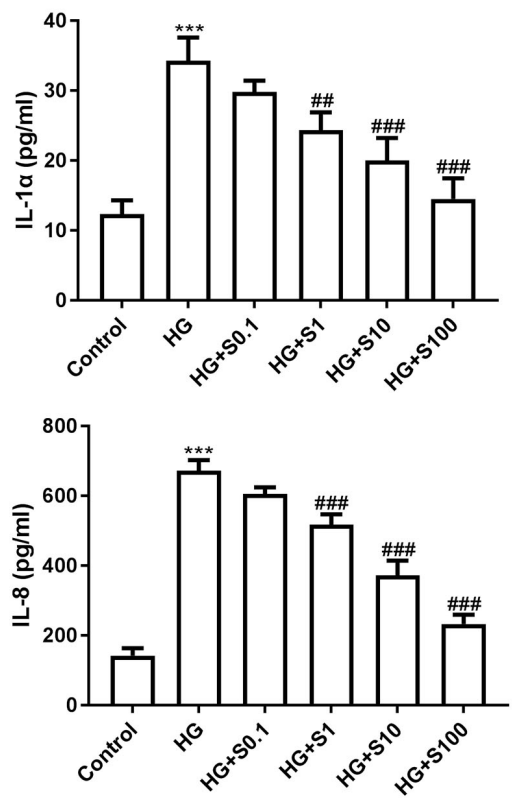

B

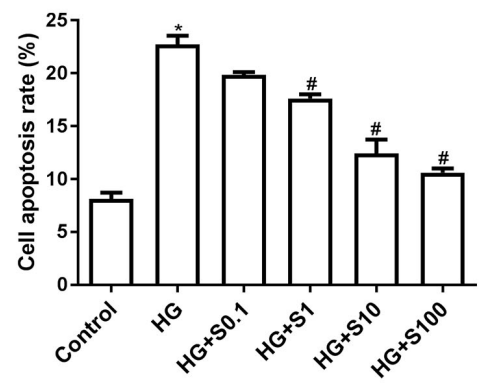

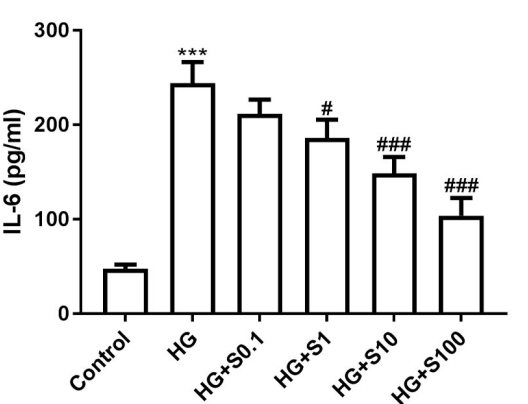

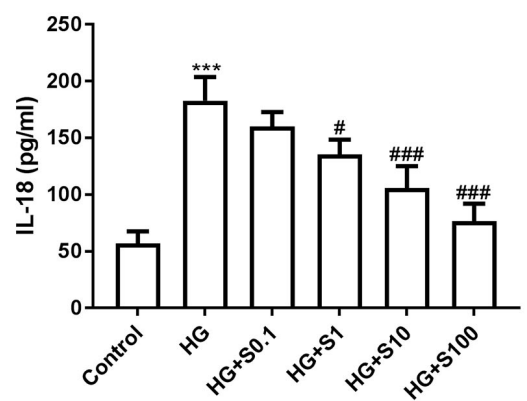

Fig. 3. Sitagliptin protected HUVECs from injuries caused by high glucose in a dose-dependent manner. Cells were treated with normal glucose, high glucose (33 mM), different concentrations of sitagliptin $(0,0.1,1,10$, and $100 \mu \mathrm{M}$ ). (A) Cell viability was detected by MTT assay. (B, C) Cell apoptosis was determined by flow cytometry. (D) The productions of IL- $1 \alpha$, IL-6, IL-8, and IL18 in HG-induced HUVECs were determined by ELISA after treated by different concentrations of sitagliptin. HUVECs, Human umbilical vein endothelial cells; $H G$, high glucose; S, sitagliptin; IL-1 $\alpha$, interleukin 1 alpha; IL-6, interleukin 6; IL-8, interleukin 8; IL-18, interleukin 18; ELISA, enzyme-linked immunosorbent assay. ${ }^{*} p<0.05$ vs. control group, ${ }^{* * *} p<0.001$ vs. control group. $" \mathrm{p}<0.05$ vs. HG group. ${ }_{\# \#} p<0.01$ vs. HG group. ${ }^{\# \#} p<0.001$ vs. HG group. 

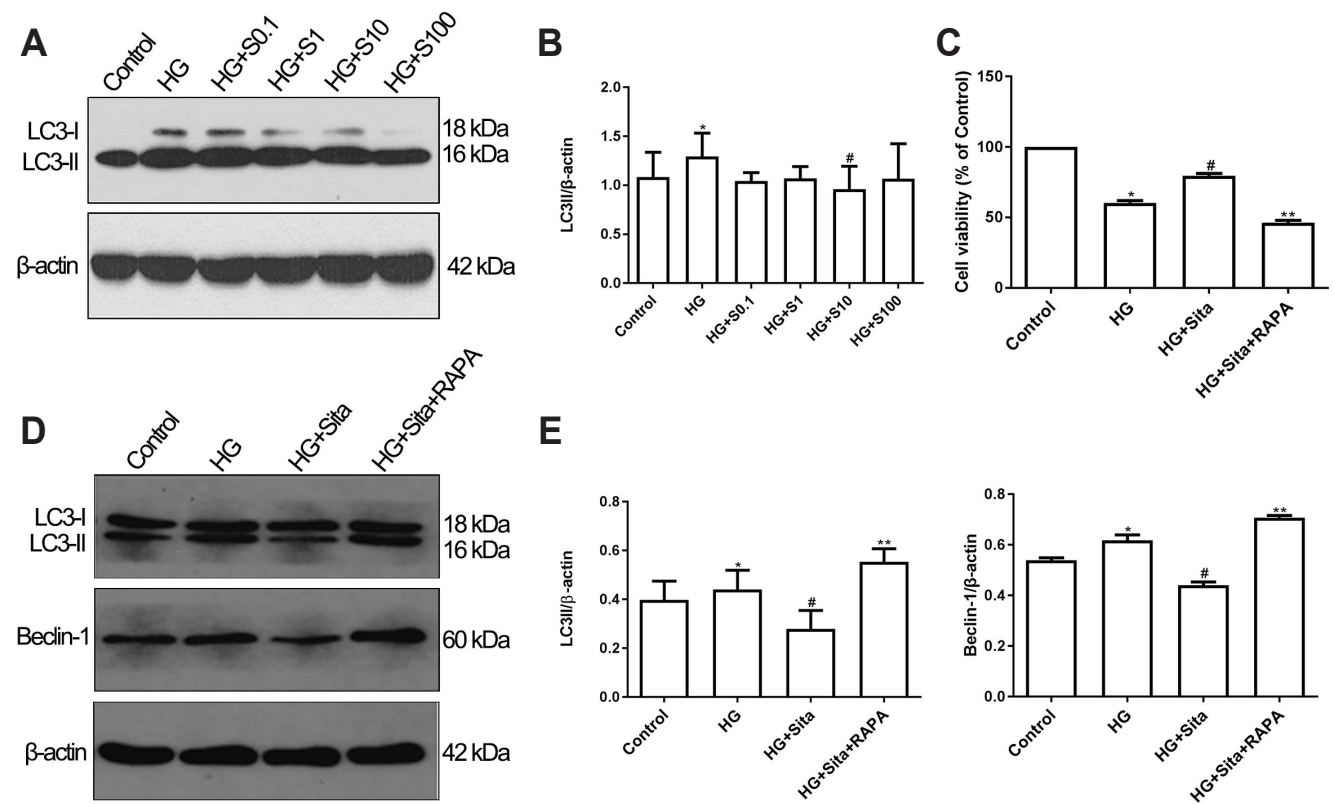

$\mathbf{E}$
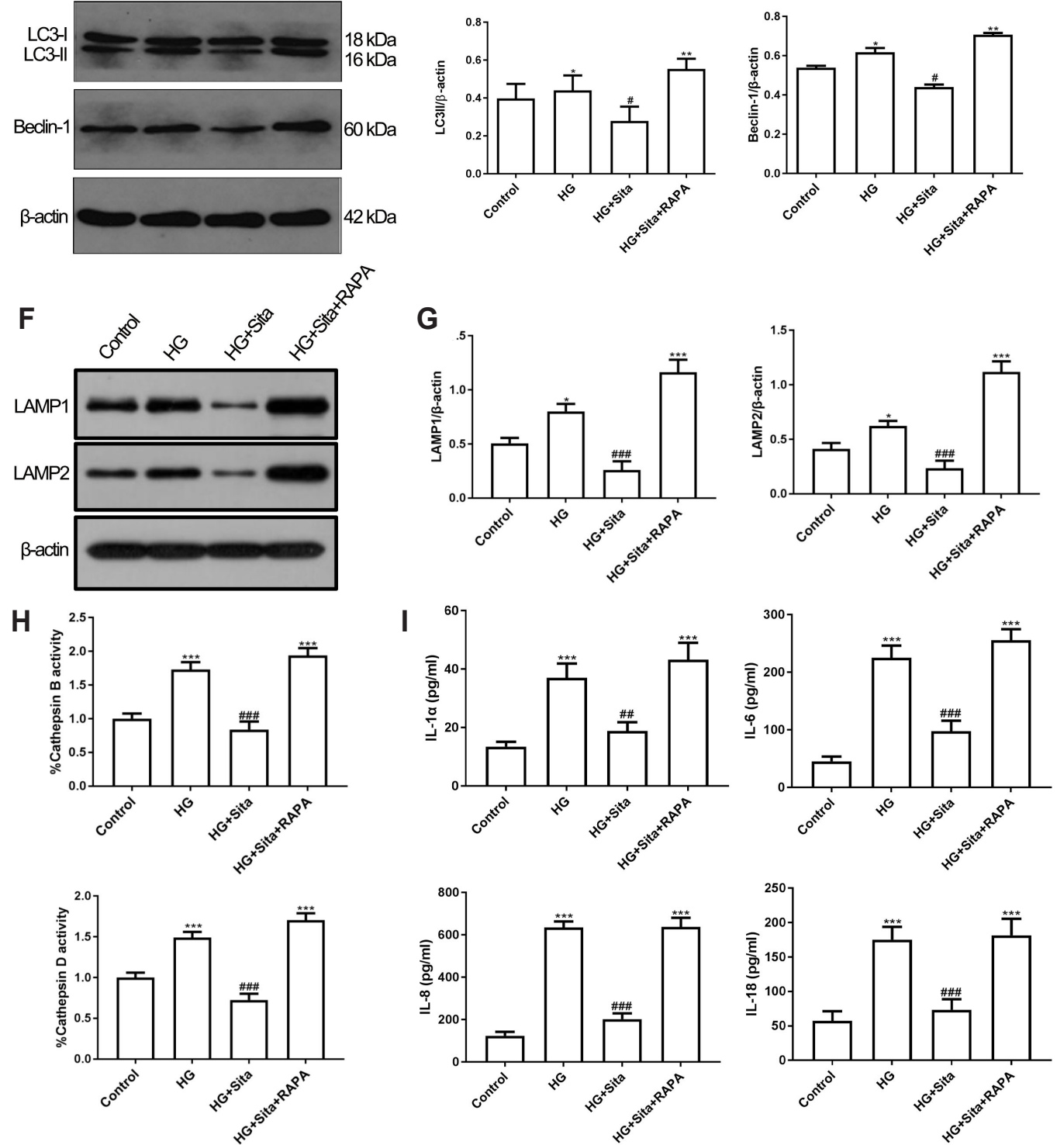

Fig. 4. Sitagliptin protected HUVECs through inhibiting over-activated autophagy. Cells were treated with normal glucose, high glucose, different concentrations of sitagliptin $(0,0.1,1,10$, and $100 \mu \mathrm{M})$. The expression of $L C 3$ was detected by Western blot (A, B). (C) Cell viability was detected after HG-induced HUVECs treated with rapamycin and sitagliptin. (D, E) Representative Western blot bands for the protein expression of LC3 and Beclin-1 in HG-induced HUVECs treated with rapamycin and sitagliptin. (F, G) Representative Western blot bands for the protein expression of LAMP1/2 in HG-induced HUVECs treated with rapamycin and sitagliptin. $(\mathrm{H})$ The Cathepsin B/D activity results of in HG-induced HUVECs treated with rapamycin and sitagliptin were presented. (I) The productions of IL-1 $\alpha$, IL-6, IL-8, IL-18 in HG-induced HUVECs were determined by ELISA after treated with rapamycin and sitagliptin. HUVECs, Human umbilical vein endothelial cells; LC3, microtubule-associated protein 1 light chain 3; HG, high glucose; S, sitagliptin; RAPA, rapamycin; LAMP1/2, lysosomal-associated membrane protein 1/2; IL-1 $\alpha$, interleukin 1 alpha; IL-6, interleukin 6; IL-8, interleukin 8; IL-18, interleukin 18; ELISA, enzyme-linked immunosorbent assay. ${ }^{*} \mathrm{p}<0.05$ vs. control group, ${ }^{* *} \mathrm{p}<0.05$ vs. HG + Sitagliptin group, ${ }^{* * *} \mathrm{p}<0.001$ vs. control group or HG + Sitagliptin group, ${ }^{\#} p<0.05$ vs. HG group, ${ }^{\# \#} \mathrm{p}<0.001$ vs. HG group, ${ }^{\# \#} \mathrm{p}<0.01$ vs. HG group. 
A

\section{A}

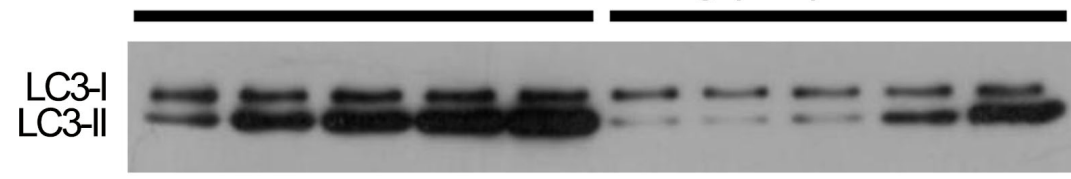

\section{Beclin-1}
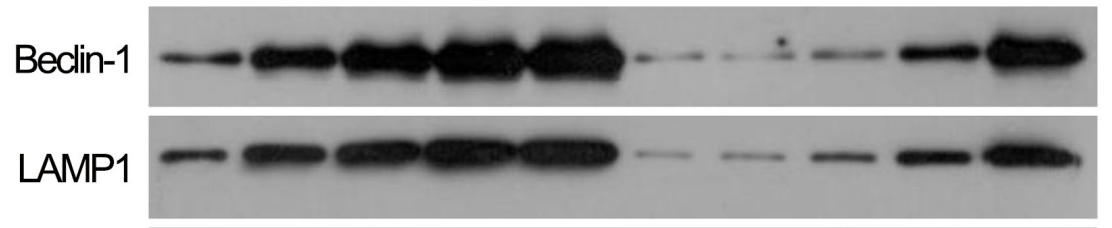

LAMP2

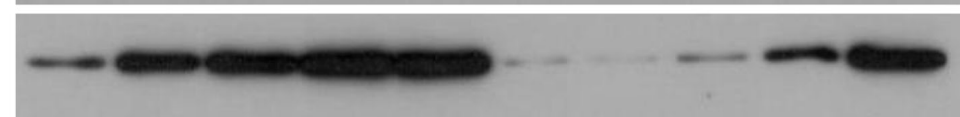

$\beta$-actin

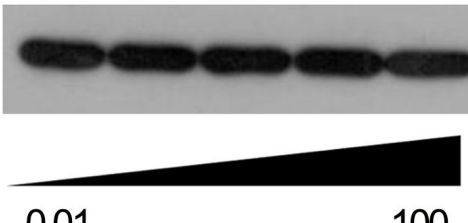

0.01

D

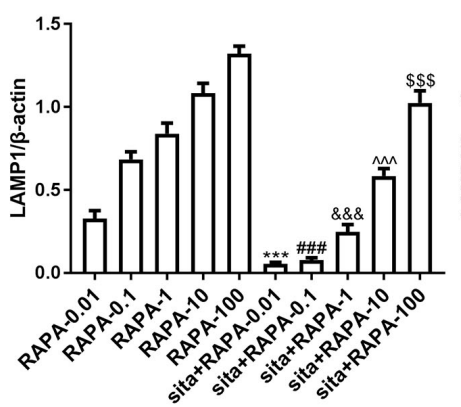

E

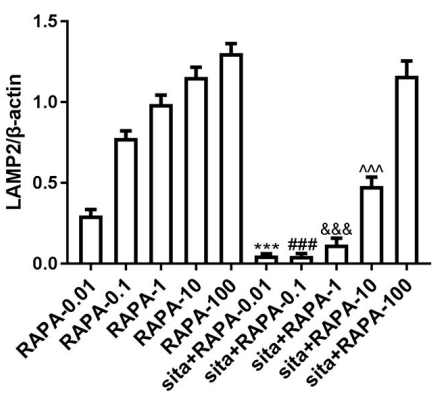

F

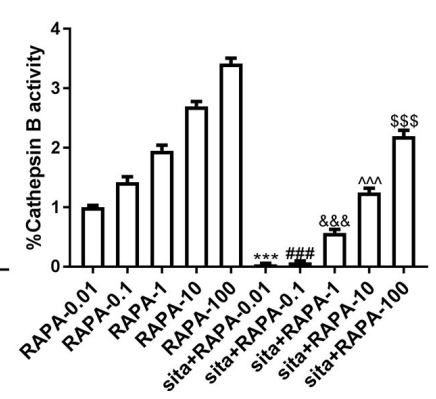

B

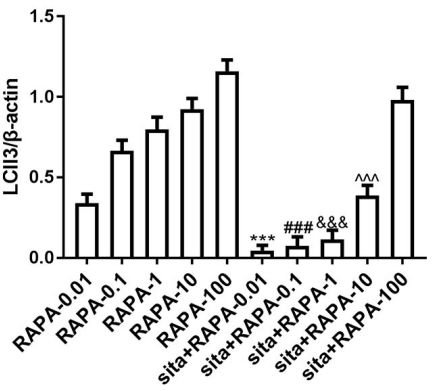

C

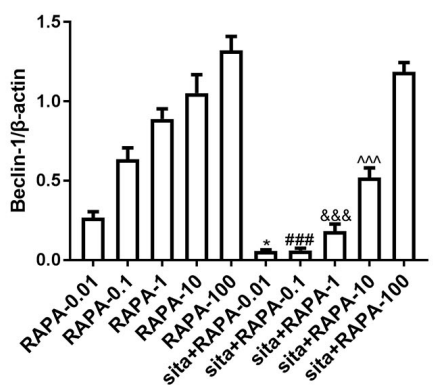

G

Fig. 5. Pretreatment with sitagliptin prevented autophagy in HUVECs exposed to rapamycin. (A-E) The protein levels of LC3, Beclin-1, LAMP1, and LAMP2 in Sitagliptin-pretreated HUVECs were determined using Western blot after exposed to rapamycin. $(F, G)$ The Cathepsin B/D activity results in Sitagliptin-pretreated HUVECs were determined after exposed to rapamycin. HUVECs, Human umbilical vein endothelial cells; LC3, microtubuleassociated protein 1 light chain 3; LAMP1/2, lysosomal-associated membrane protein 1/2. ${ }^{*} \mathrm{p}<0.05$ vs. RAPA-0.01 group. ${ }^{* * *} \mathrm{p}<0.001$ vs. RAPA-0.01 group, ${ }^{\# \# \#} \mathrm{p}<0.001$ vs. RAPA-0.1 group, ${ }^{\text {\&\&\& }} \mathrm{p}<0.001$ vs. RAPA-1 group, ${ }^{\wedge \wedge \wedge} \mathrm{p}<0.001$ vs. RAPA-10 group, ${ }^{\$ \$ \$} \mathrm{p}<0.001$ vs. RAPA-100 group.

cose increased significantly, while sitagliptin at a concentration from 1 to $100 \mu \mathrm{M}$ significantly and dose-dependently reduced high-glucose induced cell apoptosis (Fig. 3B, C). According to these results, we have chosen and used sitagliptin at the concentration of $10 \mu \mathrm{M}$ for the subsequent experiments.

Overall, our findings establish both in vivo and in vitro that sitagliptin possess an anti-apoptotic property in endothelial cells that is dose-dependent.

\section{Sitagliptin prevent autophagy in HUVEC cells exposed to high-glucose}

Next, we assessed LC3II levels in HUVECs cultured with high glucose and supplemented with sitagliptin, allowing us to systematically evaluate the impact of increasing concentrations of sitagliptin on the autophagy. The level of LC3II was significantly increased in HUVECs maintained in high glucose. Treatment with increasing concentrations of sitagliptin decreased LC3 level in a dose-dependent manner (Fig. 4A, B). To dissect the causal relationship between rapamycin-induced autophagy, and the protective role of sitagliptin, we applied rapamycin pretreatment for 30 min before the administration of high-glucose and sitagliptin. Pretreatment of cells with rapamycin, a potent inducer of autophagy, abolished the protective effect of sitagliptin on HUVECs cultured with high glucose, indicating that sitagliptin can no longer protect HUVECs from high glucose in case of an excessive autophagy (Fig. 4C-I). These results indicate that sitagliptin have a protective effect as long as an advanced autophagy (e.g., rapamycin-induced autophagy) process does not already exist.

Endothelial dysfunction is central to many inflammatory diseases affecting the vessel wall. Thus, we also assessed whether sitagliptin can interfere with cytokines released by endothelial cells exposed to glucose. As data presented on Fig. 4I shows, HG resulted in elevation in the levels of pro-inflammatory and 
chemoattractant citokines (IL-1a, IL-6, IL-8, and IL-18), while sitagliptin was able to prevent this increase, suggesting that in our STZ model sitagliptin may possess a beneficial role by modulating pro-inflammatory cytokine release upon hyperglycemia and preventing associated manifestation of the cardiovascular disease.

\section{Pretreatment with Sitagliptin prevent autophagy in HUVEC cells exposed to rapamycin}

Our findings with rapamycin pretreatment suggested that sitagliptin can be protective if administered before autophagy program is initiated (Fig. 4). To further dissect the relationship between rapamycin-induced autophagy and sitagliptin-mediated protective role, we applied sitagliptin pretreatment of HUVEC cells at $10 \mu \mathrm{M}$, then rapamycin dose $(0.01-100 \mu \mathrm{M})$ to assess at what dose sitagliptin cannot stop autophagy anymore. Then, we have assessed LC3II, Beclin-1 and LAMP1/2 as additional autophagy marker. We used no sitagliptin pre-treatment, but similar rapamycin dose treatment as control for these experiments (Fig. 5).

\section{Sitagliptin regulate autophagy via the JNK-BCl-2- Beclin-1 pathway}

Previous studies reported that sitagliptin attenuates hypoxia- induced apoptosis and autophagy in various cellular context via the JNK-Bcl-2-Beclin-1 pathway [19]. To further elucidate the molecular mechanism in endothelial cells, we tested the expression of proteins in JNK-Bcl-2-Beclin-1 pathway in vivo in our rat model. The results of western blot displayed that hyperglycemia indeed upregulated JNK and Beclin-1 expression, while Bcl-2 was downregulated in aortas of diabetic rats. Sitagliptin administration could reverse the changes in the expression of JNK, Bcl-2, and Beclin-1 (Fig. 6A-F), which suggested that sitagliptin, similar to other cell types, may regulate autophagy through JNK-Bcl2-Beclin-1 pathway in endothelial cells.

\section{DISCUSSION}

The role of chronic hyperglycemia in the development of diabetic microvascular complications has been previously established and the blood glucose lowering sitagliptin has been considered as being beneficial on endothelial function. Whether sitagliptin has any non-glycemic associated role on cardiovascular health and endothelial function remain largely unclear. Here we set up a streptozotosin-induced diabetes model that allowed us to separate the sitagliptin action on endothelial dysfunction from its blood glucose lowering function [14]. In this model beta cells

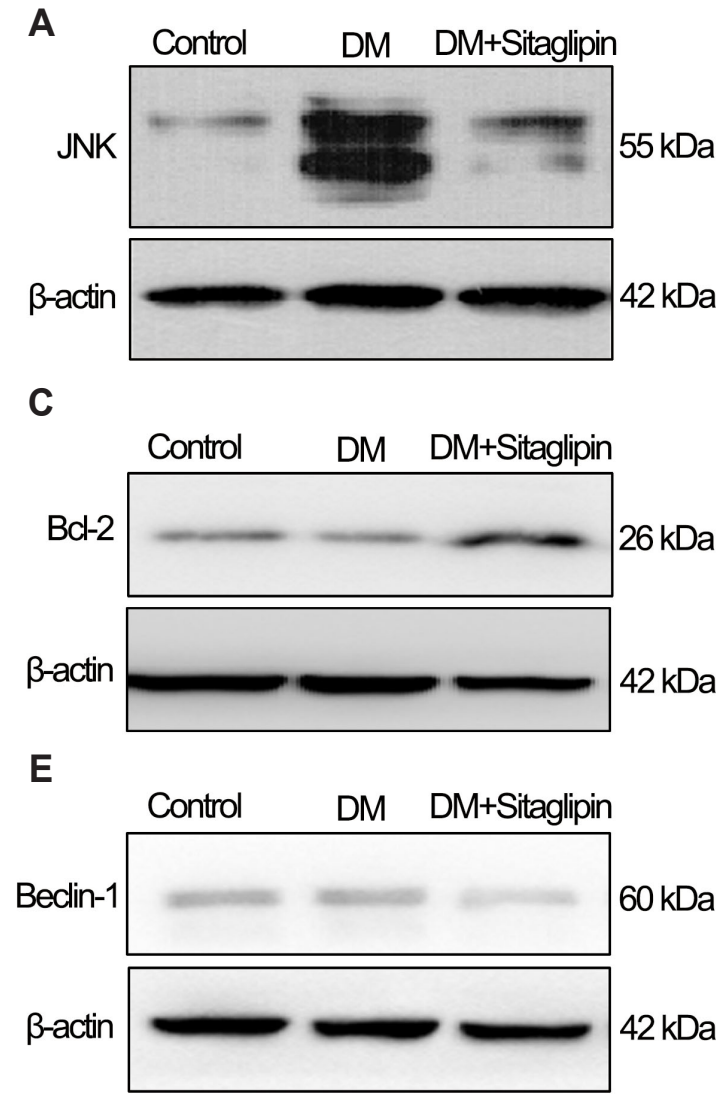

Korean J Physiol Pharmacol 2021;25(5):425-437
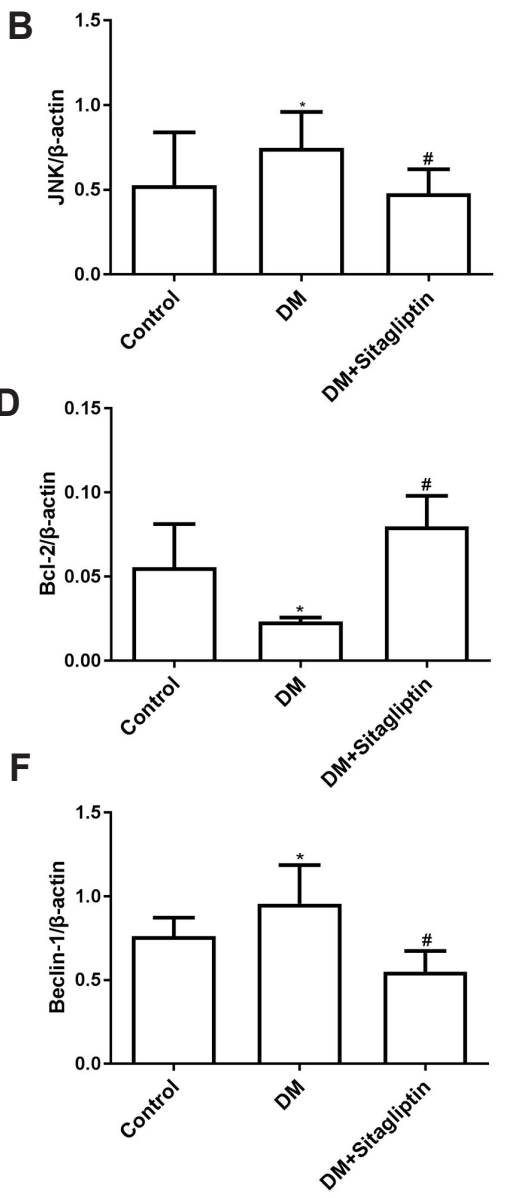

Fig. 6. Sitagliptin may regulate the expression of protein in JNK-Bcl2-Beclin-1 pathway. The expressions of JNK (A, B), BCl-2(C, D), Beclin-1(E, F) in thoracic aortas of rats in Control, DM, and $\mathrm{DM}+$ Sitagliptin groups. $\mathrm{n}=4-5$. JNK, c-Jun N-terminal Kinase; DM, diabetes mellitus. ${ }^{*} p<0.05$ vs. control group, " $p<0.05$ vs. DM group. 
function is destroyed and thus sitagliptin cannot exhibit its blood sugar lowering function. Monitoring of FBG and random blood glucose levels confirmed that our model is suitable to study the effect of non-glucose-lowering effect of the incretin-based therapy in this model. Strikingly, we found that sitagliptin was still able to reverse several pathological alterations and improve endothelial dysfunction, despite the fact that hyperglycemic conditions were not abolished.

Previous studies using sitagliptin in non-diabetic models also found that sitagliptin had protective effects on vascular endothelium, which implicated that the protective effect of sitagliptin on vascular endothelium is independent of its hypoglycemic effect $[20,21]$. Nevertheless, the improved endothelial function upon sitagliptin intervention was evident in our model, allowing us to investigate the protective role of sitagliptin at a molecular level and dissect its effect on the apoptotic and autophagy process of endothelial cell.

To ensure that our study will help to make clear conclusions on the role of sitagliptin in endothelial dysfunction and regarding the underlying cellular and molecular mechanisms, we also carried out in vitro experiments, including dose-dependent assays and multiple end-point measurements, mostly focusing on apoptotic pathway activation, as well as characterizing pro-inflmmatory cytokine release by endothelial cells in the presence or absence of sitagliptin.

Crosstalk between autophagy and apoptosis has a cardinal role in pathophysiological processes, including cardiovascular events. Understanding how a therapeutic intervention interfere with and modulate apoptosis and autophagy is a critical step in the evaluation process of a candidate drug. Our current study confirmed both in vivo and in vitro that sitagliptin play a protective role on endothelial dysfunction caused by high glucose in diabetic models. While our study is in part confirmatory, there are also important novel findings in this study. First, we document that sitagliptin can attenuate injuries of endothelial cells caused by high glucose through inhibiting excessive autophagy. Second, we demonstrate that sitagliptin regulate autophagy and apoptosis via the JNK-Bcl-2-Beclin-1 pathway in endothelial cells. Our study is particularly important as current reports suggest a contradicting model of how sitagliptin may interfere with autophagy, apoptosis and other cellular signaling pathways, leading to improved endothelial function [8].

Autophagy is a physiological and pathological process that removes long-lived proteins and damaged organelles in cells in a self-digesting manner. Although the primary role of autophagy is to maintain intracellular homeostasis and redistribute intracellular nutrients to critical physiological processes under stress, excessive autophagy can lead to cell death [22]. In vivo, our results showed that autophagy was increased in diabetic group, and the activity of autophagy was decreased after sitagliptin treatment. We speculated that excessive autophagy may be one of the risk factors of vascular endothelial injuries in diabetes, and sitagliptin can improve endothelial injuries by limiting the extent of autophagy. Thus, we assessed autophagy in vitro as well, in HUVECs. An increase in the expression of LC3II was detected in HUVECs exposed to high glucose, while sitagliptin downregulated the expression of LC3II. Beclin-1, another autophagic marker protein displayed a similar trends as LC3II, independently confirming that sitagliptin plays an anti-apoptotic role and limit excessive autophagy. In order to further elucidate the role of autophagy in the process, rapamycin, a commonly used autophagy activator, was introduced to HUVECs. At the presence of rapamycin, the protective effect of sitagliptin on endothelium was vanished, which reflected that sitagliptin played a protective role in endothelium injuries caused by high glucose.

Our findings on the anti-apoptotic and autophagy-limiting role of sitagliptin have to be interpreted in the context of available literature. Autophagy has been considered playing a role in the process of diabetic cardiovascular diseases, though the effects of autophagy is somewhat contradicting. For instance, a recent study observed that autophagy of vascular endothelial cells increased in high glucose environment, which lead to apoptosis. Consistent with our results, inhibition of autophagy in these studies could protect vascular endothelial cells from death and apoptosis [23,24]. In contrast, other studies considered autophagy as a protective factor in endothelial dysfunction and suggested that upregulation of autophagy could protect endothelium from injuries [8,25,26]. These seemingly contradicting results suggest that the role of autophagy may vary in different diabetic models [26-30], however do not explain the opposing findings in vitro cell culture models. To ensure that our observations are valid and reproducible, we repeated all the experiments at least three times independently in primary HUVECs, and carried out dose-dependent interventions with sitagliptin. Moreover, we used different end-point measurements and detection methods to confirm the findings.

Both apoptosis and autophagy are patterns of programmed cell death, which are different but correlated with each other [31]. The morphological features of apoptosis are cell volume reduction, chromatin condensation, cytoplasmic condensation, DNA degradation, and finally the formation of apoptotic bodies. Autophagy is characterized with autophagosome that forms a bilayer membrane structure, and finally forms autophagic lysosomes to achieve self-degradation. Autophagy can provide energy for cell survival, inhibits apoptosis, and also act together with apoptosis to accelerate cell death. Importantly, apoptosis and autophagy were changed in the same direction in our study, increasing at the exposure of high glucose, while decreasing after the intervention of sitagliptin.

JNK signaling pathway participating in many physiological and pathological processes in body, is closely related to autophagy and apoptosis. The activation of JNK will induce phosphorylation of $\mathrm{Bcl}-2 / \mathrm{Bcl}-\mathrm{XL}$, which promotes the separation of Beclin-1 from Beclin-1-Bcl-2/Bcl-XL complex, thus activate autophagy [32]. In our present study, sitagliptin affected the levels of JNK, Bcl-2 
and Beclin-1 proteins in aortas. However, in the absence of specific inhibitor targeted manipulation of the components of the pathway, we are not intending to conclude about the causality between JNK-Bcl-2-Beclin-1 pathway and the protective effect of sitagliptin on endothelium.

In conclusion, our study indicates that sitagliptin can improve injuries of vascular endothelial cells caused by high glucose through inhibiting excessive autophagy. Mechanistically, JNKBcl-2-Beclin-1 pathway may be an intervention target of sitagliptin to protect endothelial cells through regulating autophagy and apoptosis. Our study, along with the existing literature, provide a sight on the management of diabetic endothelial dysfunction and contribute to our understanding how incretin-based therapies may possess positive effect on cardiovascular health.

\section{ACKNOWLEDGEMENTS}

This study was funded by special fund project for clinical medical research from Chinese Medical Association (Grant No: 343) and National Natural Science Foundation of China (Grant No.81670763).

\section{CONFLICTS OF INTEREST}

The authors declare no conflicts of interest.

\section{REFERENCES}

1. An Y, Zhang P, Wang J, Gong Q, Gregg EW, Yang W, Li H, Zhang B, Shuai Y, Chen Y, Engelgau MM, Cheng Y, Hu Y, Bennett PH, Li G. Cardiovascular and all-cause mortality over a 23-year period among Chinese with newly diagnosed diabetes in the Da Qing IGT and diabetes study. Diabetes Care. 2015;38:1365-1371.

2. Mora PF, Johnson EL. Cardiovascular outcome trials of the incretinbased therapies: what do we know so far? Endocr Pract. 2017;23:8999.

3. Rizzo M, Rizvi AA, Spinas GA, Rini GB, Berneis K. Glucose lowering and anti-atherogenic effects of incretin-based therapies: GLP1 analogues and DPP-4-inhibitors. Expert Opin Investig Drugs. 2009;18:1495-1503.

4. Vilsbøll T, Garber AJ. Non-glycaemic effects mediated via GLP-1 receptor agonists and the potential for exploiting these for therapeutic benefit: focus on liraglutide. Diabetes Obes Metab. 2012;14 Suppl 2:41-49.

5. Ahrén B, Schmitz O. GLP-1 receptor agonists and DPP-4 inhibitors in the treatment of type 2 diabetes. Horm Metab Res. 2004;36:867876.

6. Wu C, Hu S, Wang N, Tian J. Dipeptidyl peptidase-4 inhibitor sitagliptin prevents high glucose-induced apoptosis via activation of AMP-activated protein kinase in endothelial cells. Mol Med Rep. 2017;15:4346-4351.
7. Tang ST, Su H, Zhang Q, Tang HQ, Wang CJ, Zhou Q, Wei W, Zhu HQ, Wang Y. Sitagliptin inhibits endothelin-1 expression in the aortic endothelium of rats with streptozotocin-induced diabetes by suppressing the nuclear factor- $\kappa \mathrm{B} / \mathrm{I} \kappa \mathrm{B} \alpha$ system through the activation of AMP-activated protein kinase. Int J Mol Med. 2016;37:15581566.

8. Wang H, Zhou Y, Guo Z, Dong Y, Xu J, Huang H, Liu H, Wang W. Sitagliptin attenuates endothelial dysfunction of Zucker diabetic fatty rats: implication of the antiperoxynitrite and autophagy. J Cardiovasc Pharmacol Ther. 2018;23:66-78.

9. Dai X, Zeng J, Yan X, Lin Q, Wang K, Chen J, Shen F, Gu X, Wang Y, Chen J, Pan K, Cai L, Wintergerst KA, Tan Y. Sitagliptin-mediated preservation of endothelial progenitor cell function via augmenting autophagy enhances ischaemic angiogenesis in diabetes. J Cell Mol Med. 2018;22:89-100.

10. Shao S, Xu Q, Yu X, Pan R, Chen Y. Dipeptidyl peptidase 4 inhibitors and their potential immune modulatory functions. Pharmacol Ther. 2020;209:107503.

11. Silva Júnior WS, Godoy-Matos AF, Kraemer-Aguiar LG. Dipeptidyl peptidase 4: a new link between diabetes mellitus and atherosclerosis? Biomed Res Int. 2015;2015:816164.

12. Price JD, Linder G, Li WP, Zimmermann B, Rother KI, Malek R, Alattar M, Tarbell KV. Effects of short-term sitagliptin treatment on immune parameters in healthy individuals, a randomized placebocontrolled study. Clin Exp Immunol. 2013;174:120-128.

13. Zilleßen P, Celner J, Kretschmann A, Pfeifer A, Racké K, Mayer P. Metabolic role of dipeptidyl peptidase 4 (DPP4) in primary human (pre)adipocytes. Sci Rep. 2016;6:23074.

14. Graham ML, Janecek JL, Kittredge JA, Hering BJ, Schuurman HJ. The streptozotocin-induced diabetic nude mouse model: differences between animals from different sources. Comp Med. 2011;61:356360 .

15. Baudin B, Bruneel A, Bosselut N, Vaubourdolle M. A protocol for isolation and culture of human umbilical vein endothelial cells. Nat Protoc. 2007;2:481-485.

16. Goncharov NV, Nadeev AD, Jenkins RO, Avdonin PV. Markers and biomarkers of endothelium: when something is rotten in the state. Oxid Med Cell Longev. 2017;2017:9759735.

17. Mizushima N, Yoshimori T. How to interpret LC3 immunoblotting. Autophagy. 2007;3:542-545.

18. Kang R, Zeh HJ, Lotze MT, Tang D. The Beclin 1 network regulates autophagy and apoptosis. Cell Death Differ. 2011;18:571-580.

19. Wang XM, Yang YJ, Wu YJ, Zhang Q, Qian HY. Attenuating hypoxia-induced apoptosis and autophagy of mesenchymal stem cells: the potential of sitagliptin in stem cell-based therapy. Cell Physiol Biochem. 2015;37:1914-1926.

20. Liu L, Liu J, Tian XY, Wong WT, Lau CW, Xu A, Xu G, Ng CF, Yao X, Gao Y, Huang Y. Uncoupling protein-2 mediates DPP-4 inhibitorinduced restoration of endothelial function in hypertension through reducing oxidative stress. Antioxid Redox Signal. 2014;21:1571-1581.

21. Zeng Y, Li C, Guan M, Zheng Z, Li J, Xu W, Wang L, He F, Xue Y. The DPP-4 inhibitor sitagliptin attenuates the progress of atherosclerosis in apolipoprotein-E-knockout mice via AMPK- and MAPK-dependent mechanisms. Cardiovasc Diabetol. 2014;13:32.

22. Levine B, Kroemer G. Autophagy in the pathogenesis of disease. Cell. 2008;132:27-42.

23. Cai X, She M, Xu M, Chen H, Li J, Chen X, Zheng D, Liu J, Chen S, 
Zhu J, Xu X, Li R, Li J, Chen S, Yang X, Li H. GLP-1 treatment protects endothelial cells from oxidative stress-induced autophagy and endothelial dysfunction. Int J Biol Sci. 2018;14:1696-1708.

24. Niu C, Chen Z, Kim KT, Sun J, Xue M, Chen G, Li S, Shen Y, Zhu Z, Wang X, Liang J, Jiang C, Cong W, Jin L, Li X. Metformin alleviates hyperglycemia-induced endothelial impairment by downregulating autophagy via the Hedgehog pathway. Autophagy. 2019;15:843-870.

25. Chen F, Chen B, Xiao FQ, Wu YT, Wang RH, Sun ZW, Fu GS, Mou Y, Tao W, Hu XS, Hu SJ. Autophagy protects against senescence and apoptosis via the RAS-mitochondria in high-glucose-induced endothelial cells. Cell Physiol Biochem. 2014;33:1058-1074.

26. Chao CL, Chuang CP, Cheng YF, Lee KR, Chang Y, Cheng SP, Chan WK, Ho FM. The protective role of autophagy in matrix metalloproteinase-mediated cell transmigration and cell death in highglucose-treated endothelial cells. Inflammation. 2016;39:830-838.

27. Zhang J, Deng H, Liu L, Liu X, Zuo X, Xu Q, Wu Z, Peng X, Ji A. $\alpha$-Lipoic acid protects against hypoxia/reoxygenation-induced injury in human umbilical vein endothelial cells through suppression of apoptosis and autophagy. Mol Med Rep. 2015;12:180-186.

28. Xu Q, Li X, Lu Y, Shen L, Zhang J, Cao S, Huang X, Bin J, Liao Y. Pharmacological modulation of autophagy to protect cardiomyocytes according to the time windows of ischaemia/reperfusion. $\mathrm{Br} J$ Pharmacol. 2015;172:3072-3085.

29. Mellor KM, Bell JR, Young MJ, Ritchie RH, Delbridge LM. Myocardial autophagy activation and suppressed survival signaling is associated with insulin resistance in fructose-fed mice. J Mol Cell Cardiol. 2011;50:1035-1043.

30. Wu X, He L, Chen F, He X, Cai Y, Zhang G, Yi Q, He M, Luo J. Impaired autophagy contributes to adverse cardiac remodeling in acute myocardial infarction. PLoS One. 2014;9:e112891.

31. Eisenberg-Lerner A, Bialik S, Simon HU, Kimchi A. Life and death partners: apoptosis, autophagy and the cross-talk between them. Cell Death Differ. 2009;16:966-975.

32. Luo S, Rubinsztein DC. BCL2L11/BIM: a novel molecular link between autophagy and apoptosis. Autophagy. 2013;9:104-105. 